\title{
The Maschke-type theorem of smash products of generalized quantum commutative algebras over weak Hopf algebras
}

\author{
Quanguo Chen
}




\title{
THE MASCHKE-TYPE THEOREM OF SMASH PRODUCTS OF GENERALIZED QUANTUM COMMUTATIVE ALGEBRAS OVER WEAK HOPF ALGEBRAS
}

\author{
Q. G. CHEN \\ Received 19 October, 2013
}

\begin{abstract}
The paper is concerned with the semisimplicity of smash products of generalized quantum commutative algebras in weak Hopf algebra setting. Let $H$ be a weak Hopf algebra over a field $k$ and $A$ any semisimple and generalized quantum commutative weak Yetter-Drinfeld $H$-module algebra. It is shown that $A \sharp H$ is semisimple if and only if $A$ is a projective left $A \sharp H$ module. Applying results to quasitriangular (weak) Hopf algebras is considered.
\end{abstract}

2010 Mathematics Subject Classification: 16W30

Keywords: weak Hopf algebra, smash product, module algebra

\section{INTRODUCTION}

In [10], Yang and Wang have proved the following statement:

Suppose that $H$ is a finite dimensional quasitriangular Hopf algebra acting on an algebra $A$ and $A$ is quantum commutative. If $A$ is semisimple, then $A \sharp H$ is semisimple if and only if $A$ is a projective left $A \sharp H$-module.

The statement above is extended to weak Hopf algebras setting by Zhai and Zhang in [11].

Weak Hopf algebras were introduced by Böhm et al. in [1] as an important generalization of ordinary Hopf algebras and groupoid algebras besides quasi-Hopf algebras, multiplier Hopf algebras, Hopf quasigroups, etc $([5,6,9])$. The axioms are the same as the ones for a Hopf algebra, except that the coproduct of the unit, the product of the counit and the antipode condition are replaced by weaker properties. The initial motivation to study weak Hopf algebras comes from the fact that some classical theory and lots of basic properties of ordinary Hopf algebras have "weak" analogues (see $[3,4,7,8]$ ).

The author was supported by the National Natural Science Foundation of China (No.11261063), the Foundation for Excelent Youth Science and Technology Innovation Talents of Xin Jiang Uygur Autonomous Region(No. 2013721043). 
Given a quasitriangular weak Hopf algebra $(H, R)$, a weak $H$-module algebra $A$ can be endowed with a suitable coaction associated to $R$ to make $A$ be a weak quantum Yetter-Drinfeld $H$-module algebra which is similar to one studied by Caenepeel et al. in the setting of Hopf algebras [2].

The purpose of this paper is to discuss the validity of the statement such as the start under the more general assumption.

The paper is organized as follows.

In Section 2, we recall basic definitions and give a summary of fundamental properties concerning weak Hopf algebras and quasitriangular weak Hopf algebras and weak Yetter-Drinfeld $H$-module algebras. In Section 3, as the main content, we discuss the semisimplicity of smash products of generalized quantum commutative algebras in weak Hopf algebra setting. Finally, the application of our results is considered.

\section{Preliminaries}

Throughout the paper $k$ is a fixed field. Unless otherwise stated, all vector spaces are over $k$ and all maps are $k$-linear. We will use the Heyneman-Sweedler notation $\Delta(c)=c_{(1)} \otimes c_{(2)}$ for coproduct (summation understood). $\otimes$ mean $\otimes_{k}$ unless otherwise specified, etc.

\subsection{Weak Hopf algebras}

Recall from Böhm et al. ([1]) that a weak Hopf algebra $(H, \Delta, \varepsilon, S)$ is both an associative algebra and a coalgebra with an antipode $S: H \rightarrow H$ satisfying the following conditions (1)-(4):

(1) $\Delta(x y)=\Delta(x) \Delta(y)$ for all $x, y \in H$;

(2) $\Delta^{2}(1)=(\Delta(1) \otimes 1)(1 \otimes \Delta(1)), \quad \Delta^{2}(1)=(1 \otimes \Delta(1))(\Delta(1) \otimes 1)$;

(3) $\varepsilon(x y z)=\varepsilon\left(x y_{(1)}\right) \varepsilon\left(y_{(2)} z\right), \varepsilon(x y z)=\varepsilon\left(x y_{(2)}\right) \varepsilon\left(y_{(1)} z\right)$ for all $x, y, z \in H$;

(4) $x_{(1)} S\left(x_{(2)}\right)=\varepsilon\left(1_{(1)} x\right) 1_{(2)}, S\left(x_{(1)}\right) x_{(2)}=1_{(1)} \varepsilon\left(x 1_{(2)}\right)$,

$$
S\left(x_{(1)}\right) x_{(2)} S\left(x_{(3)}\right)=S(x) \text {, for all } x \in H .
$$

Let $H$ be a weak Hopf algebra. Then we define the maps $\varepsilon_{t}, \varepsilon_{s}: H \rightarrow H$ by the formulas

$$
\varepsilon_{t}(x)=\varepsilon\left(1_{(1)} x\right) 1_{(2)}, \quad \varepsilon_{s}(x)=1_{(1)} \varepsilon\left(x 1_{(2)}\right)
$$

and denote by $H_{t}$ the image $\varepsilon_{t}(H)$ and denote by $H_{s}$ the image $\varepsilon_{s}(H)$.

Let $H$ be a weak Hopf algebra. The following results hold from Böhm et al. ([1]), for all $h, g \in H$,

(W1) $H_{t}$ and $H_{s}$ are two sub-algebras of $H$,

(W2) $\Delta(1)=1_{(1)} \otimes 1_{(2)} \in H_{s} \otimes H_{t}, \quad \varepsilon_{t}(h) \varepsilon_{s}(g)=\varepsilon_{s}(g) \varepsilon_{t}(h)$,

(W3) $\Delta\left(\varepsilon_{t}(h)\right)=1_{(1)} \varepsilon_{t}(h) \otimes 1_{(2)}, \quad \Delta\left(\varepsilon_{s}(g)\right)=1_{(1)} \otimes \varepsilon_{S}(g) 1_{(2)}$,

$(\mathrm{W} 4) h_{(1)} \otimes \varepsilon_{S}\left(h_{(2)}\right)=h 1_{(1)} \otimes S\left(1_{(2)}\right), \quad \varepsilon_{t}\left(h_{(1)}\right) \otimes h_{(2)}=S\left(1_{(1)}\right) \otimes 1_{(2)} h$, 
$\left(\right.$ W5) $h_{(1)} \otimes \varepsilon_{t}\left(h_{(2)}\right)=1_{(1)} h \otimes 1_{(2)}, \quad \varepsilon_{s}\left(h_{(1)}\right) \otimes h_{(2)}=1_{(1)} \otimes h 1_{(2)}$,

(W6) $\varepsilon_{t} \circ \varepsilon_{t}=\varepsilon_{t}, \quad \varepsilon_{s} \circ \varepsilon_{s}=\varepsilon_{s}$,

(W7) $\varepsilon_{t} \circ S=\varepsilon_{t} \circ \varepsilon_{S}=S \circ \varepsilon_{S}, \quad \varepsilon_{S} \circ S=\varepsilon_{S} \circ \varepsilon_{t}=S \circ \varepsilon_{t}$,

(W8) $S(h g)=S(g) S(h), S\left(h_{(2)}\right) \otimes S\left(h_{(1)}\right)=S(h)_{(1)} \otimes S(h)_{(2)}$ and

$S(1)=1, \varepsilon \circ S=\varepsilon$,

(W9) $h_{1} \varepsilon_{S}(g) \otimes h_{2}=h_{(1)} \otimes h_{(2)} S\left(\varepsilon_{S}(g)\right), h_{1} \otimes \varepsilon_{t}(g) h_{2}=S\left(\varepsilon_{t}(g)\right) h_{(1)} \otimes h_{(2)}$.

\subsection{Quasitriangular weak Hopf algebras}

Recall from Nikshych et al. in [8] that a quasitriangular weak Hopf algebra is a pair $(H, R)$, where $H$ is a weak Hopf algebra and $R=R^{1} \otimes R^{2} \in \Delta^{o p}(1)(H \otimes$ $H) \Delta(1)$ such that

(Q1) There exists $\bar{R} \in \Delta(1)\left(H \otimes_{k} H\right) \Delta^{o p}(1)$ with $R \bar{R}=\Delta^{o p}(1)$ and $\bar{R} R=\Delta(1)$.

(Q2) For all $h \in H$, we have

$$
\left\{\begin{array}{l}
\Delta^{o p}(h) R=R \Delta(h) \\
(i d \otimes \Delta) R=R_{13} R_{12} \\
(\Delta \otimes i d) R=R_{13} R_{23}
\end{array}\right.
$$

where $\Delta^{o p}$ denotes the comultiplication opposite to $\Delta, R_{12}=R \otimes 1, R_{23}=1 \otimes R$, etc., as usual.

Let $(H, R)$ be a quasitriangular weak Hopf algebra. Then the following six identities hold:

$$
\left\{\begin{array}{l}
\left(\varepsilon_{s} \otimes i d\right)(R)=\Delta(1), \quad\left(i d \otimes \varepsilon_{s}\right)(R)=(S \otimes i d) \Delta^{o p}(1), \\
\left(\varepsilon_{t} \otimes i d\right)(R)=\Delta^{o p}(1), \quad\left(i d \otimes \varepsilon_{t}\right)(R)=(S \otimes i d) \Delta(1), \\
(S \otimes i d)(R)=\left(i d \otimes S^{-1}\right)(R)=\bar{R}, \quad(S \otimes S)(R)=R
\end{array}\right.
$$

\subsection{Weak module algebras}

Let $H$ be a weak Hopf algebra. An algebra $A$ is called a left weak $H$-module algebra, if $A$ is a left $H$-module via $h \otimes a \mapsto h \cdot a$ such that, for any $a, b \in A$ and $h \in H$,

$$
h \cdot(a b)=\left(h_{(1)} \cdot a\right)\left(h_{(2)} \cdot b\right), \quad h \cdot 1_{A}=\varepsilon_{t}(h) \cdot 1_{A} .
$$

Let $H$ be a weak Hopf algebra and $A$ a weak left $H$-module algebra. Recall from Nikshych in [8] that a weak smash product $A \sharp H$ of $A$ and $H$ is defined on a $k$-vector space $A \otimes_{H_{t}} H$, where $H$ is a left $H_{t}$-module via its multiplication and $A$ is a right $H_{t}$ - module via

$$
a \cdot x=S^{-1}(x) \cdot a=a\left(x \cdot 1_{A}\right), a \in A, x \in H_{t} .
$$

Its multiplication is given by the following formula:

$$
(a \sharp h)(b \sharp g)=a\left(h_{(1)} \cdot b\right) \sharp h h_{(2)} g,
$$

for any $a, b \in A$ and $g, h \in H$. Then $A \sharp H$ is an associative algebra with unit $1_{A} \sharp 1_{H}$. 


\subsection{Weak quantum Yetter-Drinfeld $H$-module algebras}

A $k$-algebra $A$ is called a weak quantum Yetter-Drinfeld $H$-module algebra, if $A$ satisfies the following conditions:

(WQ1) $A$ is a weak left $H$-module algebra,

(WQ2) $A$ is a right $H^{o p}$-comodule algebra, i.e., the comodule structure map

$$
\rho: A \rightarrow A \otimes H \text { satisfies }
$$

$a_{[0]} \otimes a_{[1]}=1_{(1)} \cdot a_{[0]} \otimes 1_{(2)} a_{[1]}, \rho(a b)=a_{[0]} b_{[0]} \otimes b_{[1]} a_{[1]}, \rho(1)=\left(i d \otimes \varepsilon_{t}\right) \rho(1)$, where $\rho(a)=a_{[0]} \otimes a_{[1]}$ denotes the coaction.

(WQ3) (WQ1) and (WQ2) satisfy the Yetter-Drinfeld condition

$$
\left(h_{(2)} \cdot a\right)_{[0]} \otimes\left(h_{(2)} \cdot a\right)_{[1]} h_{(1)}=h_{(1)} \cdot a_{[0]} \otimes h_{(2)} a_{[1]},
$$

for all $h \in H, a \in A$.

Remark 1. The Yetter-Drinfeld condition is equivanlent to

$$
\rho(h \cdot a)=h_{(2)} \cdot a_{[0]} \otimes h_{(3)} a_{[1]} S^{-1}\left(h_{(1)}\right) .
$$

Example 1. Let $H$ be a weak Hopf algebra. $\left\{1_{(1)} h S\left(1_{(2)}\right) \mid h \in H\right\}$ is a weak quantum Yetter-Drinfeld $H$-module algebra with the action and coaction given as follows:

$g \cdot\left(1_{(1)} h S\left(1_{(2)}\right)\right)=g_{(1)} h S\left(g_{(2)}\right), \rho\left(1_{(1)} h S\left(1_{(2)}\right)\right)=1_{(1)} h_{(2)} S\left(1_{(2)}\right) \otimes S^{-1}\left(h_{(1)}\right)$.

Example 2. Let $(H, R)$ be a quasitriangular weak Hopf algebra. Given any left weak $H$-module algebra $A$, one can define a right $H^{o p}$-coaction on $A$ as follows:

$$
\rho(a)=R^{2} \cdot a \otimes R^{1} .
$$

With the above coaction, it is easily checked that $A$ is a weak quantum Yetter-Drinfeld $H$-module algebra.

\section{THE MAIN RESULTS}

In this section, we assume that $H$ is a weak Hopf algebra with bijective antipode $S$, and $A$ a weak left $H$-module algebra, and $A \sharp H$ the weak smash product algebra.

Definition 1. Let $H$ be a weak Hopf algebra and $A$ a weak quantum YetterDrinfeld $H$-module algebra. $A$ is called a generalized quantum commutative algebras, if $A$ satisfies, for all $a, b \in H$

$$
a b=b_{[0]}\left(b_{[1]} \cdot a\right) .
$$

Lemma 1. For any weak quantum Yetter-Drinfeld $H$-module algebra A. Then, for all $a \in A$,

$$
\begin{aligned}
1_{[0]} \otimes 1_{[1](1)} \otimes 1_{[1](2)} & =1_{[0]} \otimes 1_{(1)} 1_{[1]} \otimes 1_{(2)} \\
a_{[0]} \otimes \varepsilon_{t}\left(a_{[1]}\right) & =a 1_{[0]} \otimes 1_{[1]},
\end{aligned}
$$




$$
a_{[0]} \otimes \varepsilon_{S}\left(a_{[1]}\right)=1_{[0]} a \otimes S\left(1_{[1]}\right) .
$$

Lemma 2. For any generalized quantum commutative algebra A. Then, for all $a, b \in A$,

$$
a b=\left(S\left(a_{[1]}\right) \cdot b\right) a_{[0]} \Longleftrightarrow a b=b_{[0]}\left(b_{[1]} \cdot a\right) .
$$

Proof. For all $a, b \in A$, we have

$$
\begin{aligned}
\left(S\left(a_{[1]}\right) \cdot b\right) a_{[0]} & =a_{[0][0]}\left(a_{[0][1]} S\left(a_{[1]}\right) \cdot b\right) \\
& =a_{[0]}\left(\varepsilon_{t}\left(a_{[1]}\right) \cdot b\right) \\
& =a 1_{[0]}\left(1_{[1]} \cdot b\right)=a b .
\end{aligned}
$$

Conversely, For all $a, b \in A$, we have

$$
\begin{aligned}
b_{[0]}\left(b_{[1]} \cdot a\right) & =\left(S\left(b_{[0][1]}\right) b_{[1]} \cdot a\right) b_{[0][0]} \\
& =\left(S\left(b_{[1](1)}\right) b_{[1](2)} \cdot a\right) b_{[0]} \\
& =\left(S\left(1_{[1]}\right) \cdot a\right) 1_{[0]} b=a b .
\end{aligned}
$$

So we finish the proof.

Lemma 3. For any left weak $H$-module algebra $A$. Then $M$ is a left $A \sharp H$-module if and only if $M$ is both a left $H$-module and a left A-module and satisfies the following compatible condition

$$
h \cdot(a \cdot m)=\left(h_{(1)} \cdot a\right) \cdot\left(h_{(2)} \cdot m\right),
$$

for all $h \in H, a \in A$ and $m \in M$.

Lemma 4. Let $H$ be a weak Hopf algebra and $A$ a generalized quantum commutative algebra. Then, for all $a, b \in A$,

$$
a_{[0]} b_{[0]} \otimes b_{[1]} a_{[1]}=b_{[0]}\left(b_{[1](2)} \cdot a\right)_{[0]} \otimes\left(b_{[1](2)} \cdot a\right)_{[1]} b_{[1](1)} .
$$

Proof. For all $a, b \in A$, apply $\rho$ to the identity $a b=b_{[0]}\left(b_{[1]} \cdot a\right)$, we have

$$
\begin{aligned}
\rho\left(b_{[0]}\left(b_{[1]} \cdot a\right)\right) & =b_{[0][0]}\left(b_{[1]} \cdot a\right)_{[0]} \otimes\left(b_{[1]} \cdot a\right)_{[1]} b_{[0][1]} \\
& =b_{[0]}\left(b_{[1](2)} \cdot a\right)_{[0]} \otimes\left(b_{[1](2)} \cdot a\right)_{[1]} b_{[1](1)} \\
& =\rho(a b) .
\end{aligned}
$$

The proof is completed.

Lemma 5. Let $H$ be a weak Hopf algebra and $A$ a generalized quantum commutative algebra. If $M$ is a left $A \sharp H$-module, then $M$ is an A-bimodule with the right module action of $A$ on $M$ as follows

$$
\left\llcorner: M \otimes A \rightarrow M, m \otimes a \mapsto m\left\llcorner a=a_{[0]} \cdot\left(a_{[1]} \cdot m\right),\right.\right.
$$

and for all $h \in H$,

$$
h \cdot(m \angle a)=\left(h_{(1)} \cdot m\right)<\left(h_{(2)} \cdot a\right) .
$$


Proof. First, we shall check that $M$ is a right $A$-module. In fact, for all $m \in M$ and $a, b \in A$, we have

$$
\begin{aligned}
(m \angle a) \angle b & =\left(a_{[0]} \cdot\left(a_{[1]} \cdot m\right)\right)<b \\
& =b_{[0]} \cdot\left(b_{[1]} \cdot\left(a_{[0]} \cdot\left(a_{[1]} \cdot m\right)\right)\right) \\
& =b_{[0]} \cdot\left(\left(b_{[1](1)} \cdot a_{[0]}\right) \cdot\left(b_{[1](2)} a_{[1]} \cdot m\right)\right) \\
& =\left(b_{[0]}\left(b_{[1](2)} \cdot a\right)_{[0]}\right) \cdot\left(\left(b_{[1](2)} \cdot a\right)_{[1]} b_{[1](1)} \cdot m\right) \\
& =a_{[0]} b_{[0]} \cdot\left(b_{[1]} a_{[1]} \cdot m\right) \\
& =(a b)_{[0]}\left((a b)_{[1]} \cdot m\right) \\
& =m \angle a b \\
m \angle 1 & =1_{[0]} \cdot\left(1_{[1]} \cdot m\right) \\
& =1_{[0]}\left(1_{(1)} \cdot 1_{A}\right) \cdot\left(1_{(2)} 1_{[1]} \cdot m\right) \\
& =1_{[0]}\left(S\left(1_{(1)}\right) \cdot 1_{A}\right) \cdot\left(1_{(2)} \varepsilon_{t}\left(1_{[1]}\right) \cdot m\right) \\
& =\left(1_{[0]}\left(\varepsilon_{t}\left(1_{[1]}\right) S\left(1_{(1)}\right) \cdot 1_{A}\right)\right) \cdot\left(1_{(2)} \cdot m\right) \\
& =\left(1_{[0]}\left(1_{[1]} S\left(1_{(1)}\right) \cdot 1_{A}\right)\right) \cdot\left(1_{(2)} \cdot m\right) \\
& =\left(S\left(1_{(1)}\right) \cdot 1_{A}\right) \cdot\left(1_{(2)} \cdot m\right) \\
& =\left(1_{(1)} \cdot 1_{A}\right) \cdot\left(1_{(2)} \cdot m\right)=m .
\end{aligned}
$$

Now, we shall check $M$ is an $A$-bimodule, i.e., $(a \cdot m)<b=a \cdot(m<b)$. As a matter of fact,

$$
\begin{aligned}
(a \cdot m) \_b & =b_{[0]} \cdot\left(b_{[1]} \cdot(a \cdot m)\right) \\
& =b_{[0]} \cdot\left(\left(b_{[1](1)} \cdot a\right) \cdot\left(b_{[1](2)} \cdot m\right)\right) \\
& =\left(b_{[0]}\left(b_{[1](1)} \cdot a\right)\right) \cdot\left(b_{[1](2)} \cdot m\right) \\
& =\left(b_{[0][0]}\left(b_{[0][1]} \cdot a\right)\right) \cdot\left(b_{[1]} \cdot m\right) \\
& =a b_{[0]} \cdot\left(b_{[1]} \cdot m\right) \\
& =a \cdot(m \angle b) .
\end{aligned}
$$

Finally, for all $a \in A, m \in M$ and $h \in H$,

$$
\begin{aligned}
\left(h_{(1)} \cdot m\right) \leftarrow\left(h_{(2)} \cdot a\right) & =\left(h_{(2)} \cdot a\right)_{[0]}\left(\left(h_{(2)} \cdot a\right)_{[1]} h_{(1)} \cdot m\right) \\
& =\left(h_{(1)} \cdot a_{[0]}\right)\left(h_{(2)} a_{[1]} \cdot m\right) \\
& =h \cdot\left(a_{[0]} \cdot\left(a_{[1]} \cdot m\right)\right)=h \cdot(m-a) .
\end{aligned}
$$

The proof is completed. 
Lemma 6. For all left $A \sharp H$-module $M$, we have

$$
A \sharp H \operatorname{Hom}(A, M) \cong M^{H}, F: f \mapsto f\left(1_{A}\right),
$$

where $M^{H}=\left\{m \in M \mid h \cdot m=\varepsilon_{t}(h) \cdot m, \quad \forall h \in H\right\}$ and $A$ is left $A \sharp H$-module via

$$
(a \sharp h) \cdot b=a(h \cdot b) \text {. }
$$

Proof. For given $0 \neq m \in M^{H}$, we define a map via

$$
f: a \mapsto\left(a \sharp 1_{H}\right) \cdot m \text {. }
$$

Throughout standard computation, we can show that $f \in \in_{A \sharp H} \operatorname{Hom}(A, M)$. Based on this, we can check that $F$ is bijective in a straightforward way.

Lemma 7. Let $H$ be a weak Hopf algebra and $A$ a generalized quantum commutative algebra. Then, for all left $A \sharp H$-modules $M$ and $N$.

(1) $\operatorname{Hom}_{A}\left(M_{A}, N_{A}\right) \in \in_{A \sharp H} M$,

(2) $\operatorname{Hom}_{A}\left(M_{A}, N_{A}\right)^{H}={ }_{A \sharp H} \operatorname{Hom}(M, N)$,

where $\operatorname{Hom}_{A}\left(M_{A}, N_{A}\right)$ denotes the space of the right A-module homomorphisms.

Proof. (1) Let $M, N \in_{A \sharp H} M$. Then $M, N$ are both $A$-bimodules from Lemma 5. For all $a \sharp h \in A \sharp H, f \in \operatorname{Hom}_{A}\left(M_{A}, N_{A}\right) \in \in_{A \sharp H} M$, define action of $A \sharp H$ on $\operatorname{Hom}_{A}\left(M_{A}, N_{A}\right) \in_{A \sharp H} M$ by

$$
((a \sharp h) \cdot f)(m)=a \cdot\left(h_{(1)} \cdot f\left(S\left(h_{(2)}\right) \cdot m\right)\right),
$$

for all $m \in M$. We shall check $(a \sharp h) \cdot f \in \operatorname{Hom}_{A}\left(M_{A}, N_{A}\right)$. For all $c \in A$, we have

$$
\begin{aligned}
((a \sharp h) \cdot f)(m \angle c) & =a \cdot\left(h_{(1)} \cdot f\left(S\left(h_{(2)}\right) \cdot(m \angle c)\right)\right) \\
& =a \cdot\left(\left(h_{(1)} \cdot f\left(S\left(h_{(4)}\right) \cdot m\right)\right)<h_{(2)} S\left(h_{(3)}\right) \cdot c\right) \\
& =a \cdot\left(\left(1_{(1)} h_{(1)} \cdot f\left(S\left(h_{(2)}\right) \cdot m\right)\right)<1_{(2)} \cdot c\right) \\
& =a \cdot\left(\left(h_{(1)} \cdot f\left(S\left(h_{(2)}\right) \cdot m\right)\right)<c\right) \\
& =\left(a \cdot\left(h_{(1)} \cdot f\left(S\left(h_{(2)}\right) \cdot m\right)\right)\right)<c \\
& =((a \sharp h) \cdot f)(m) \angle c .
\end{aligned}
$$

It is checked directly that $\operatorname{Hom}_{A}\left(M_{A}, N_{A}\right) \in_{A \sharp H} M$ is a left $A \sharp H$-module.

(2) For all $f \in_{A \sharp H} \operatorname{Hom}(M, N)$, then $f$ is both a left $H$-module morphism and a left $A$-module morphism between $M$ and $N$. We shall check that $f \in$ $\operatorname{Hom}_{A}\left(M_{A}, N_{A}\right)^{H}$. For all $m \in M$ and $a \in A$, since

$$
f\left(m\llcorner a)=f\left(a_{[0]} \cdot\left(a_{[1]} \cdot m\right)\right)=a_{[0]} \cdot\left(a_{[1]} \cdot f(m)\right)=f(m)\llcorner m,\right.
$$

we conclude that $f$ is a right $A$-linear. Also, for any $h \in H$ and $m \in M$, we have

$$
(h \cdot f)(m)=h_{(1)} \cdot f\left(S\left(h_{(2)}\right) \cdot m\right)=h_{(1)} S\left(h_{(2)}\right) \cdot f(m)=\varepsilon_{t}(h) \cdot f(m),
$$

i.e., $f \in \operatorname{Hom}_{A}\left(M_{A}, N_{A}\right)^{H}$. Conversely, First, we can define the left $H_{S}$-action on $M$ by restricting the $A \sharp H$-action on $M: x \cdot m=m\left\llcorner\left(h \cdot 1_{A}\right)\right.$, for all $m \in M, h \in H_{s}$. 
Using Remark 1 and Lemma 3, it is easy to see that right $A$-module homomorphisms are morphisms of left $H_{S}$-modules. For all $f \in \operatorname{Hom}_{A}\left(M_{A}, N_{A}\right)^{H}$, we have

$$
\begin{aligned}
h \cdot f(m) & =h_{(1)} \varepsilon_{S}\left(h_{(2)}\right) \cdot f(m) \\
& =h_{(1)} \cdot f\left(\varepsilon_{S}\left(h_{(2)}\right) \cdot m\right) \\
& =\left(h_{(1)} \cdot f\right)\left(h_{(2)} \cdot m\right) \\
& =\left(\varepsilon_{t}\left(h_{(1)}\right) \cdot f\right)\left(h_{(2)} \cdot m\right) \\
& =\left(\varepsilon_{t}\left(1_{(1)}\right) \cdot f\right)\left(1_{(2)} h \cdot m\right) \\
& =\left(1_{(1)} \cdot f\right)\left(1_{(2)} h \cdot m\right) \\
& =1_{(1)} \cdot f\left(S\left(1_{(2)}\right) 1_{(3)} h \cdot m\right) \\
& =1_{(1)} \varepsilon_{S}\left(1_{(2)}\right) \cdot f(h \cdot m)=f(h \cdot m),
\end{aligned}
$$

i.e., $f$ is a left $H$-module map. Now, we shall check that $f$ is a left $A$-module map. Indeed, for all $a \in A$ and $m \in M$,

$$
\begin{aligned}
f(a \cdot m) & =f\left(a_{[0]} \cdot\left(1_{[1]} \cdot m\right)\right) \\
& =f\left(a_{[0]} \cdot\left(\varepsilon_{t}\left(a_{[1]}\right) \cdot m\right)\right) \\
& =f\left(a_{[0]} \cdot\left(a_{[1] 1} S\left(a_{[1] 2}\right) \cdot m\right)\right) \\
& =f\left(a_{[0][0]} \cdot\left(a_{[0][1]} S\left(a_{[1]}\right) \cdot m\right)\right) \\
& =f\left(\left(S\left(a_{[1]}\right) \cdot m\right)<a_{[0]}\right) \\
& =\left(S\left(a_{[1]}\right) \cdot f(m)\right)<a_{[0]}=a \cdot f(m) .
\end{aligned}
$$

So we get that $f \in \in_{A \sharp H} \operatorname{Hom}(M, N)$.

Now, we can present the main result in this section.

Theorem 1. Let $H$ be a weak Hopf algebra and $A$ a generalized quantum commutative. If $A$ is semisimple, then $A \sharp H$ is semisimple if and only if $A$ is a projective left $A \sharp H$-module algebra.

Proof. Assume $A$ is a projective left $A \sharp H$-module, then the functor $A \sharp H \operatorname{Hom}(A,-)$ is exact. For any left $A \sharp H$-module $M$, it is viewed as a right $A$ module via " $\angle$ " in Lemma 5. Since $A$ is semisimple, $M$ is projective as a right $A$ module. Hence the functor $\operatorname{Hom}_{A}(M,-)$ is exact. Further, the composition functor $A \sharp H \operatorname{Hom}\left(A, \operatorname{Hom}_{A}(M,-)\right)$ is also exact. From Lemma 6 and 7 , we get

$$
A \sharp H \operatorname{Hom}\left(A, \operatorname{Hom}_{A}(M, N)\right) \cong_{A \sharp H} \operatorname{Hom}(M, N),
$$

for any left $A \sharp H$-module $M$ and $N$. Then $M$ is a projective left $A \sharp H$-module, hence $A \sharp H$ is semisimple. The converse is obvious.

Next, we shall apply Theorem 1 to Example 1 . Given a quasitriangular weak Hopf algebra $(H, R)$ and weak $H$-module algebra $A, A$ is a weak quantum Yetter-Drinfeld 
$H$-module algebra with the coaction defined in Example 1. Then the generalized quantum commutative condition in Definition 1 takes the following form

$$
a b=\left(R^{2} \cdot b\right)\left(R^{1} \cdot a\right) .
$$

With the assumption above and by Theorem 1, we have the main result of Zhai and Zhang in [11].

Corollary 1. Let $(H, R)$ be a quasitriangular weak Hopf algebra and A a quantum commutative. If $A$ is semisimple, then $A \sharp H$ is semisimple if and only if $A$ is a projective left $A \sharp H$-module algebra.

Remark 2. If $\Delta(1)=1 \otimes 1$, weak Hopf algebras are just Hopf algebras. Corollary 1 recovers to the results of Yang and Wang in [10].

\section{ACKNOWLEDGEMENT}

The author would like to thank the referee for his/her valuable comments on this article.

\section{REFERENCES}

[1] G. Böhm, F. Nill, and K. Szlachányi, "Weak Hopf algebras i: integral theory and $C^{*}$-sructure,” $J$. Alg., vol. 221, pp. 385-438, 1999.

[2] S. Caenepeel, F. Van Oystaeyen, and Y. Zhang, "Quantum Yang-Baxter module algebras," $K$ Theory, vol. 8, pp. 231-255, 1994.

[3] P. Etingof and D. Nikshych, "Dynamical quantum groups at roots of 1," Duke J. Math., vol. 108, pp. 135-168, 2001.

[4] P. Etingof and O. Schiffmann, "Lectures on the dynamical Yang-Baxter equations," Lect. Notes Lond. Math. Soc., vol. 290, pp. 89-129, 2001.

[5] Z. M. Jiao and Y. L. Wang, "The smash coproduct for Hopf quasigroups," Int. Electron. J. Alg., vol. 12, pp. 94-102, 2012.

[6] J. Klim and S. Majid, "Hopf quasigroups and the algebraic 7-sphere," J. Alg., vol. 323, pp. 30673110, 2010.

[7] D. Nikshych, "A duality theorem for quantum groupoids," Contemporary Mathematics, vol. 267, pp. 237-243, 2000.

[8] D. Nikshych, V. Turaev, and L. Vainerman, "Quantum groupoids and invariants of knots and 3manifolds," Topol. Appl., vol. 127, pp. 91-123, 2003.

[9] A. Van Daele, "Multiplier Hopf algebras," Trans. Amer. Math. Soc., vol. 342, pp. 917-932, 1994.

[10] S. L. Yang and Z. X. Wang, "The semisimplicity of smash products of quantum commutatitive algebras," Comm. Alg., vol. 27, pp. 1165-1170, 1999.

[11] W. J. Zhai and L. Y. Zhang, "The Maschke's theorem for smash products of quasitriangular weak Hopf algebras," Abh. Math. Sem. Hambg., vol. 81, pp. 35-44, 2011.

Author's address

Q. G. Chen

Quan-guo Chen, Yili Normal University, Department of Mathematics, 835000 Yining, China

E-mail address: cqg211@163.com 